\section{Transcarinal needle aspiration in the diagnosis of mediastinal adenitis in a patient infected with the human immunodeficiency virus}

G Julià Serdà, F Rodriguez de Castro, J M Fernandez Sánchez-Alarcos, J Caminero Luna, F Diaz López, P Cabrera Navarro

\begin{abstract}
Tuberculous mediastinal lymphadenopathy in a patient infected with human immunodeficiency virus (HIV) was diagnosed by means of transcarinal needle aspiration via a fibreoptic bronchoscope.
\end{abstract}

Extrapulmonary and disseminated forms of tuberculosis are frequent among HIV positive patients. ${ }^{1-3}$ Diagnosis of mediastinal adenitis is particularly difficult, given the inaccessibility of this region, and usually requires a surgical approach. We report the diagnosis of tuberculous infection of mediastinal glands by the use of a flexible transbronchial needle through a fibreoptic bronchoscope.

\section{Case report}

A 37 year old man, with a one month history of cough, fever, reduced appetite and weight loss, was admitted to hospital. He was a 20 pack year smoker and had a history of parenteral drug abuse. Hepatitis was diagnosed at the age of 30 . On examination the patient was pale and thin, with soft, white patches on the tonsils and tongue. His temperature was $38^{\circ} \mathrm{C}$, pulse rate 120 beats/min, respiratory rate $25 /$ $\mathrm{min}$, and blood pressure $100 / 60 \mathrm{~mm} \mathrm{Hg}$. He had mild expiratory wheezes in both lungs but no crackles. No lymphadenopathy was present and the rest of the clinical examination showed nothing abnormal.

Abnormal laboratory results included normocytic anaemia (haemoglobin concentration $11 \cdot 1 \mathrm{~g} / \mathrm{dl}$ ) and a white blood cell count of $10.1 \times 10^{9} / 1(86 \%$ neutrophils, $4 \%$ band forms, $6 \%$ lymphocytes, and $4 \%$ monocytes). The erythrocyte sedimentation rate was 65 $\mathrm{mm}$ in the first hour. The Mantoux test (PPD, $5 \mathrm{TU}$ ) produced a $10 \mathrm{~mm}$ induration. A chest radiograph showed mediastinal widening with a normal lung appearance. Computed tomography of the thorax showed multiple enlarged mediastinal lymph nodes, up to $2 \mathrm{~cm}$ in diameter (figure). Microscopic examination of sputum specimens stained by the Ziehl-Neelsen and fluorescence methods showed no acid fast bacilli. Specimens taken from an oropharyngeal exudate disclosed Candida albicans. A test for antibodies to the human immunodeficiency virus gave a positive result.

On the third day in hospital fibreoptic bronchoscopy showed external compression of the carina and subcarinal widening. No endobronchial lesion was seen. Transbronchial aspiration with a 22 gauge type IA
Pulmonary Division, Nuestra Señora del Pino Hospital, Las Palmas de Gran Canaria, Spain G Julià Serdà F Rodriguez de Castro J M Fernandez SánchezAlarcos

J Caminero Luna

F Diaz López

P Cabrera Navarro

Address for reprint requests: Dr Gabriel Julià Serdà, Sección de Neumologia, Hospital Nuestra Señora de Pino, Angel Guimerá 93, 35005 Las Palmas de Gran Canaria, Spain

Accepted 12 December 1989

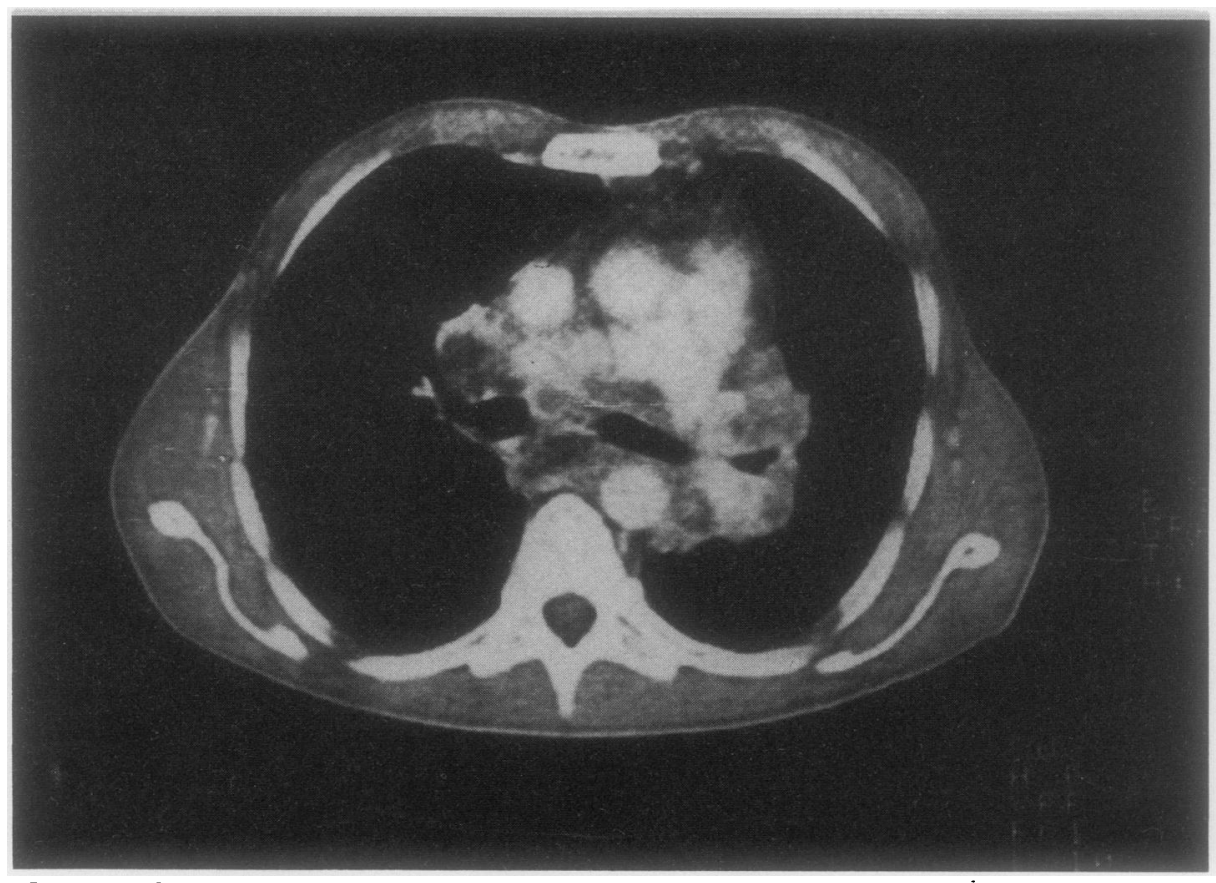

Contrast enhanced computed tomogram of the chest, showing enlargement of left and right hilar lymph nodes and posterior subcarinal lymph nodes. 
transbronchial needle (Mill-Rose Company, Mentor, Ohio) was performed at the anterior and posterior aspects of the main carina. ${ }^{4}$ Thirty millilitres of purulent fluid was aspirated. Cytological examination gave negative results. Ziehl-Neelsen staining showed many acid fast bacilli. No complications occurred after the procedure. The patient started antituberculous treatment, with slow resolution of his symptoms. Specimen culture in Lowenstein-Jensen media grew Mycobacterium tuberculosis.

\section{Discussion}

The differential diagnosis of mediastinal masses with no abnormality in the lung is an important clinical problem. One of the most important causes of mediastinal widening on a chest radiograph is lymph node enlargement, usually due to tumour or infection. Lymphadenopathy is one of the commonest forms of extrapulmonary tuberculosis, especially in children and young adults, though enlargement of mediastinal lymph nodes is an unusual presentation of tuberculosis in non-immunosuppressed white adults. ${ }^{5}$

Patients infected with HIV have clinical and radiological patterns that differ from those of reactive tuberculosis in non-HIV patients in the following respects: (1) hilar or mediastinal adenopathy (or both) is common and is probably part of a generalised lymphatic tuberculosis; (2) pulmonary infiltrates occur with about equal frequency in the upper and lower lung fields and do not cavitate or heal with scars; (3) disseminated disease is reported in a high percentage of HIV positive patients. These differences between HIV and non-HIV patients are almost certainly related to differences in immunity. ${ }^{1-3}$ Abnormalities in the mediastinum are traditionally investigated by mediastinoscopy, anterior mediastinotomy, or thoracotomy. Wang developed transbronchial needle aspiration via a fibreoptic bronchoscope in 1983, to overcome the need for more invasive procedures in the diagnosis of cancer. ${ }^{6}$ The procedure is useful for sampling paratracheal, subcarinal, and hilar regions and it also has therapeutic potential. ${ }^{6-8} \mathrm{We}$ are unaware of any previous report in which transbronchial needle aspiration has been used in the diagnosis of infection in the mediastinum. ${ }^{9}$ With this technique we obtained specimens from our patient that facilitated rapid, safe, and accurate diagnosis of tuberculosis, thereby avoiding more aggressive procedures and a risk of the transmission of HIV to the thoracic surgeon.

1 Rieder HL, Snider DE. Tuberculosis and the acquired immunodeficiency syndrome. Chest 1986;90:469-70.

2 Soriano E, Mallolas J, Gatell JM, et al. Characteristics of tuberculosis in HIV-infected patients: a case-control study. AIDS 1988;2:429-32.

3 Selwyn PA, Hartel D, Lewis VA, et al. A prospective study of the risk of tuberculosis among intravenous drug users with human immunodeficiency virus infection. $N$ Engl J with human immunodef

4 Shure D, Fedullo PF. The role of transcarinal needle aspiration in the staging of bronchogenic carcinoma. Chest 1984;86:693-6.

5 Hopewell PC. Mycobacterial diseases. In: Murray JF, Nadel JA, eds. Textbook of respiratory medicine. Philadelphia: Saunders, 1988:856-915.

6 Wang KP, Terry PB. Transbronchial needle aspiration in the diagnosis and staging of bronchogenic carcinoma. Am Rev Respir Dis 1983;127:344-7.

7 Wang KP, Nelson S, Scatarige J, Siegelman S. Transbronchial needle aspiration of a mediastinal mass: therapeutic implications. Thorax 1983;38:556-7.

8 Schwartz AR, Fishman EK, Wang KP. Diagnosis and treatment of a bronchogenic cyst using transbronchial needle aspiration. Thorax 1986;41:326-7.

9 Gay PC, Brutinel MW. Transbronchial needle aspiration in practice of bronchoscopy. Mayo Clin Proc 1989;64: $158-62$. 